\title{
MODEL CALCULATIONS OF PLASTIC DEFORMATION OF TWO-PHASE MATERIALS WITH FIBRE STRUCTURE
}

\author{
H. J. BUNGE and W. BÖCKER \\ Department of Physical Metallurgy, Technical University of Clausthal, Germany
}

\begin{abstract}
Plastic flow in two-phase fibre composites during axially symetric deformation is modelled assuming homogeneous deformation of the fibres of the harder phase and inhomogeneous flow in the softer matrix. The distribution of flow between fibres and matrix is described by the deformation ratio $\alpha$ which follows from the assumption of minimum deformation work. The main features of the model agree quite well with experimental results obtained in several powder metallurgically prepared two-phase composites.
\end{abstract}

KEY WORDS: Two-phase materials, plastic deformation, model calculations.

\section{INTRODUCTION}

Plastic deformation of single phase polycrystalline materials has been studied experimentally in an enormous number of investigations. Theoretical model calculations have also been done, following several mathematical models. It has been found that models assuming homogeneous strains in the individual crystallite as well as in the aggregate, e.g. the Taylor model, are already capable of explaining plastic anisotropy and texture formation in a quite reasonable approximation. In more sophisticated models inhomogeneities of stress and strain from grain to grain and even within a grain are taken into account. The deformation of polyphase materials has also been studied in many investigations (see e.g. Liesner and Wassermann 1969, Wahl and Wassermann 1970, Frommeyer 1976, Wassermann 1976).

A comprehensive review of the deformation behaviour of two-phase materials was given by Fischmeister and Karlsson 1977. It turned out that the behaviour of two-phase materials can be understood sufficiently in terms of a generalized "rule of mixture" considering the individual deformation state of both phases (see e.g. Werner and Stüwe 1985, Ankam and Margolin 1986, Funkenbusch, Lee and Courtney 1987, Cho and Gurland 1988, Lian, Jiang and Liu 1991, Poech and Fischmeister 1992). Particularly, texture development in two-phase materials was found to depend essentially on the relative yield stress difference of the phases. From texture measurements it was concluded that the internal deformation degree of the harder phase is smaller than that of the material as a whole. In some cases it could further be concluded that the harder phase could assume a deviating deformation mode, e.g. nearly plane strain in an axially deformed compositite. This leads, for instance, to the curling effect of iron particles in a copper matrix and to a deviating texture type of the iron particles. Although 
the softer phase must carry a higher part of the macroscopic strain, its texture is often found to be much weaker. This has been attributed to flow inhomogeneities in the matrix around the particles of the harder phase.

Plastic deformation of two-phase materials can be studied for virtually all materials combinations in all composition ranges using powder metallurgical methods. In a recent study (Böcker and Bunge 1988a,b, Böcker 1992), metal composites were prepared by powder mixing and extrusion, followed by profile rolling and wire drawing up to total deformation degrees of $\eta=99.99 \%$ in the composition range from 0 to $100 \%$. During extrusion the stresses were measured. The fibre deformation degrees were obtained by quantitative metallographic methods. Furthermore, the inhomogeneity of flow was assessed from the shapes of the phase particles. Neutron diffraction texture measurements were used to get information about the deformation of matrix and fibres.

The mechanical behaviour of the composites as well as the participation of the fibres in the overall deformation could be understood quite well within a rather simple model assuming homogeneous deformation of the harder fibres and inhomogeneous flow of the matrix. The shape changes of the fibre particles as well as the texture changes of the matrix were not yet taken into consideration.

\section{FORMULATION OF THE MODEL}

We consider a fibre composite consisting of a softer matrix in which stronger fibres are embedded. The volume fraction of the fibres be $x_{F}$, that of the matrix be $x_{M}$. It is

$$
x_{F}+x_{M}=1
$$

We assume axially symmetric, and macroscopically homogeneous deformation of the composite with a strain tensor

$$
\varepsilon_{\mathrm{ij}}=\varepsilon \cdot\left[\begin{array}{ccc}
-0.5 & 0 & 0 \\
0 & -0.5 & 0 \\
0 & 0 & 1
\end{array}\right]
$$

at a macroscopic tensile stress

$$
\sigma_{i j}=\sigma \cdot\left[\begin{array}{lll}
0 & 0 & 0 \\
0 & 0 & 0 \\
0 & 0 & 1
\end{array}\right]
$$

Deformation of the fibres is also assumed to be axially symmetric and homogeneous but with a deviating strain

$$
\varepsilon_{i j}^{F}=\varepsilon_{F} \cdot\left[\begin{array}{ccc}
-0.5 & 0 & 0 \\
0 & -0.5 & 0 \\
0 & 0 & 1
\end{array}\right]
$$

We introduce the deformation ratio $\alpha$ by

$$
\varepsilon_{F}=\alpha \cdot \varepsilon
$$


The stresses in the fibres are also assumed to be homogeneous tensile stresses

$$
\sigma_{i j}^{F}=\sigma_{F} \cdot\left[\begin{array}{lll}
0 & 0 & 0 \\
0 & 0 & 0 \\
0 & 0 & 1
\end{array}\right]
$$

The deformation of the matrix is assumed to be inhomogeneous. The strain field in the matrix can be expressed by the homogeneous average $\overline{\varepsilon_{i j}^{M}}$ and a local deviator term

$$
\varepsilon_{i j}^{M}(\vec{r})=\overline{\varepsilon_{i j}^{M}}+\Delta \varepsilon_{i j}^{M}(\vec{r})
$$

The average strain has the form

$$
\overline{\varepsilon_{i j}^{M}}=\varepsilon_{M} \cdot\left[\begin{array}{ccc}
-0.5 & 0 & 0 \\
0 & -0.5 & 0 \\
0 & 0 & 1
\end{array}\right]
$$

whereas the integral over the deviator term vanishes

$$
\int_{M} \Delta \varepsilon_{i j}^{M}(\vec{r}) d V=0
$$

Similarly, the stresses in the matrix are expressed by

with

$$
\sigma_{i j}^{M}(\vec{r})=\overline{\sigma_{i j}^{M}}+\Delta \sigma_{i j}^{M}(\vec{r})
$$

$$
\overline{\sigma_{i j}^{M}}=\sigma^{M} \cdot\left[\begin{array}{lll}
0 & 0 & 0 \\
0 & 0 & 0 \\
0 & 0 & 1
\end{array}\right]
$$

and

$$
\int_{M}{ }_{i j} \rightarrow \Delta \sigma^{M}(r) d V=0
$$

The strain of the composite is the integral over the local strains

$$
\varepsilon_{i j}=\int_{M} \varepsilon_{i j}^{M}(\vec{r}) d V+\int_{F} \varepsilon_{i j}^{F} d V
$$

from which follows with eq $(4,7,9)$

$$
\varepsilon_{i j}=x_{M} \cdot \overline{\varepsilon_{i j}^{M}}+x_{F} \cdot \varepsilon_{i j}^{F}
$$

and with eq $(2,4,8)$

$$
\varepsilon=x_{M} \cdot \varepsilon_{M}+x_{F} \cdot \varepsilon_{F}
$$


with eq (5) it follows

$$
\varepsilon_{M}=\varepsilon \cdot \frac{1-\alpha \cdot x_{F}}{x_{M}}
$$

We define the "deformation misfit" between matrix and fibres

$$
\Delta e=\frac{\varepsilon_{M}}{\varepsilon}-\frac{\varepsilon_{F}}{\varepsilon}=\frac{1-\alpha}{x_{M}}
$$

We make the assumption that both the deviator terms of strain $\Delta \varepsilon_{i j}^{M}(\vec{r})$ and stress $\Delta \sigma_{i j}^{M}(\vec{r})$ are proportional to the deformation misfit $\Delta e$ between matrix and fibres

$$
\begin{aligned}
& \Delta \varepsilon_{i j}^{M}(\vec{r})=\Delta e \cdot \varepsilon \cdot \varphi_{i j}(\vec{r}) \\
& \Delta \sigma_{i j}^{M}(\vec{r})=\Delta e \cdot \sigma^{M} \cdot \Psi_{i j}(\vec{r})
\end{aligned}
$$

where $\varphi_{i j}(\vec{r})$ and $\psi_{i j}(\vec{r})$ are dimensionless functions of the location $\vec{r}$ which depend on the geometry of the fibres and their distribution in the composite.

The energy dissipation rate is given by

$$
W=\int_{\mathrm{v}} \sigma_{i j}(\vec{r}) \cdot \varepsilon_{i j}(\vec{r}) d V
$$

This can be split into the terms of the matrix and the fibres respectively

$$
W=W_{M}+W_{F}
$$

where

$$
\begin{aligned}
& W=\varepsilon \cdot \sigma \\
& W_{F}=x_{F} \cdot \sigma_{F} \cdot \varepsilon_{F} \\
& W_{M}=x_{M} \cdot \sigma_{M} \cdot \varepsilon_{M}+\Delta e^{2} \cdot \sigma_{M} \cdot \varepsilon \cdot \emptyset
\end{aligned}
$$

with

$$
\emptyset=\int_{M} \varphi_{i j}(\vec{r}) \cdot \Psi_{i j}(\vec{r}) d V
$$

with eqs (5), (16), (17) one obtains

$$
\begin{aligned}
& W_{F}=x_{F} \cdot \sigma_{F} \cdot \alpha \cdot \varepsilon \\
& W_{M}=x_{M} \cdot \sigma_{M} \cdot \frac{1-\alpha \cdot x_{F}}{x_{M}} \cdot \varepsilon+\left[\frac{1-\alpha}{x_{M}}\right]^{2} \sigma_{M} \cdot \emptyset \cdot \varepsilon
\end{aligned}
$$


The macroscopic stress $\sigma$ can then the expressed in the form

$$
\sigma=\frac{W}{\varepsilon}=x_{F} \cdot \sigma_{F} \cdot \alpha+\sigma_{M} \cdot\left(1-\alpha \cdot x_{F}\right)+\frac{\emptyset \cdot \sigma_{M} \cdot(1-\alpha)^{2}}{x_{M}^{2}}
$$

It is assumed that the composite deforms with a minimum of deformation work i.e. at a given strain $\varepsilon$ with the minimum of external stress with respect to the deformation ratio $\alpha$ of the fibres

$$
\sigma(\alpha)=\operatorname{Min}
$$

This leads to the condition

$$
x_{F} \cdot \sigma_{F}-x_{F} \cdot \sigma_{M}-2 \frac{\emptyset \cdot \sigma_{M}}{x_{M}^{2}}(1-\alpha)=0
$$

from which the deformation ratio $\alpha$ is obtained

$$
\alpha=1-\frac{\sigma_{F}-\sigma_{M}}{\sigma_{M}} \cdot \frac{x_{F} \cdot x_{M}^{2}}{2 \varnothing}
$$

We introduce the yield stress ratio

$$
\rho=\frac{\sigma_{F}}{\sigma_{M}}
$$

of matrix and fibres. With this quantity, the deformation ratio $\alpha$ can be expressed in the form

$$
\alpha=1-(\rho-1) \cdot \frac{x_{F} \cdot x_{M}^{2}}{2 \varnothing}
$$

For further analysis of eq (33) the dependence of the strain inhomogeneity function $\emptyset$ on the volume fraction of fibres $x_{F}$ must be known. This requires numerical calculations of the stress and strain distribution in the composite as they were done, for instance by Durand and Pastor 1992, Canova, Wenk and Molinari 1992, Neti, Vijayshankar and Ankam 1991, McHugh, Asaro and Shih 1993, for specific assumed distributions of the phases. In the present model we shall, however, restrict ourselves to some plausibility considerations. At first we assume in this model that inhomogeneities of $\sigma_{i j}$ and $\varepsilon_{i j}$ in the matrix are only due to the two-phase nature of the composite. The single phase matrix without fibres is assumed to deform homogeneously. For small volume fractions of fibres it is then reasonable to assume that $\varnothing\left(x_{F}\right)$ is proportional to $x_{F}$. Furthermore, the integral in eq (25) is to be taken over the volume of the matrix. Therefore we assume here that for small volume fractions of the matrix $\emptyset$ is proportional to the volume fraction $x_{M}$ of the matrix. Hence, we put

$$
\varnothing\left(x_{F}\right)=x_{F} \cdot x_{M} \cdot \chi\left(x_{F}\right)
$$


whereby $\chi\left(x_{F}\right)$ is another unknown function of $x_{F}$ and the geometry of the fibres and their distribution in the matrix. As a further approximation we will consider, later on, the case that $\chi\left(x_{F}\right)$ is constant

$$
\chi\left(x_{F}\right)=\text { const. }=\chi_{0}
$$

With eq (34), eq (33) takes on the form

$$
\alpha=1-(\rho-1) \cdot \frac{1-x_{F}}{2 \cdot \chi\left(x_{F}\right)} \text { for } \rho \leq \rho_{\text {crit }}
$$

This expression results from the minimum, if condition eq (29) based on eq $(26,27)$. Strictly speaking, the above considerations are only valid for

$$
\alpha \geq 0
$$

Otherwise, the fibres would contract when the composite is elongated. In this case in eq (26). $\alpha$ would have to be replaced by $-\alpha$. The minimum of eq $(26,27)$ is then at

$$
\alpha=0 \text { for } \rho>\rho_{\text {crit }}
$$

The critical value of $\rho$ above which the fibres do not deform is obtained by putting the right-hand side of eq (36) to zero

$$
\rho_{c r i t}\left(x_{F}\right)=\frac{2 \cdot \chi\left(x_{F}\right)}{1-x_{F}}+1
$$

With the definition of $\chi\left(x_{F}\right)$ of eq (34) the flow stress of the composite eq (28) takes on the form

$$
\sigma=\sigma_{M}+\alpha \cdot x_{F} \cdot \sigma_{M}(\rho-1)+\sigma_{M} \cdot \frac{x_{F} \cdot \chi\left(x_{F}\right)}{x_{M}} \cdot(1-\alpha)^{2}
$$

Expressing $\alpha$ by the two minimum values eq (36) and eq (38) one obtains the flow stress ratio of the composite compared to that of the matrix

$$
\left.\frac{\sigma}{\sigma_{M}}=1+x_{F} \cdot(\rho-1) \cdot(1-(\rho-1)) \cdot \frac{1-x_{F}}{4 \cdot \chi\left(x_{F}\right)}\right) \quad \text { for } \rho \leq \rho_{\text {crit }}
$$

and

$$
\frac{\sigma}{\sigma_{M}}=1+\frac{x_{F} \cdot \chi\left(x_{F}\right)}{1-x_{F}} \quad \text { for } \rho \geq \rho_{\text {crit }}
$$

In this latter case the flow stress of the composite is, of course, independent of the yield stress ratio $\rho$. 
The main results of this model are contained in eq $(36,38)$ giving the deformation ratio $\alpha$ of the harder fibres compared to the total deformation of the composite and in eq $(41,42)$ giving the relative flow stress of the composite compared to that of the pure matrix. Furthermore, eq (39) distinguishes the two cases of deforming and non-deforming fibres. Within the framework of this model the function $\chi\left(x_{F}\right)$ remains unknown. This function considers the stress-strain field in the matrix. The present model does not allow to determine this function. Therefore, the first approximation eq (35) will be given particular consideration. Comparison with experimental data obtained from several composite systems for $x_{F} \rightarrow 0$ gave a value for $\chi_{0}$ (Figure 5) which is then the only free parameter in this approximation. It was found

$$
\chi_{0}=2,5
$$

With this value and eq (35) the deformation ratio $\alpha$ as a function of $\rho$ was plotted in Figure 1 with the volume fraction $x_{F}$ of fibres as a parameter. Similarly, Figure 2 gives $\alpha$ as a function of the volume fraction $x_{F}$ with $\rho$ as a parameter. Figure 3 gives the yield stress ratio of the composite as a function of the volume fraction of fibres with $\rho$ as a parameter. The field of the $\rho$-dependent curves is limited by the "boundary curve" (eq 42) representing the case of undeformed fibres. It is seen, in agreement with Figure 1, that below $\rho=6$ the fibres always are deformed whereas above $\rho=6$ the fibres take part in the deformation only above a critical volume fraction. Finally, the critical $\rho$-value, eq (39), is given in Figure 4.

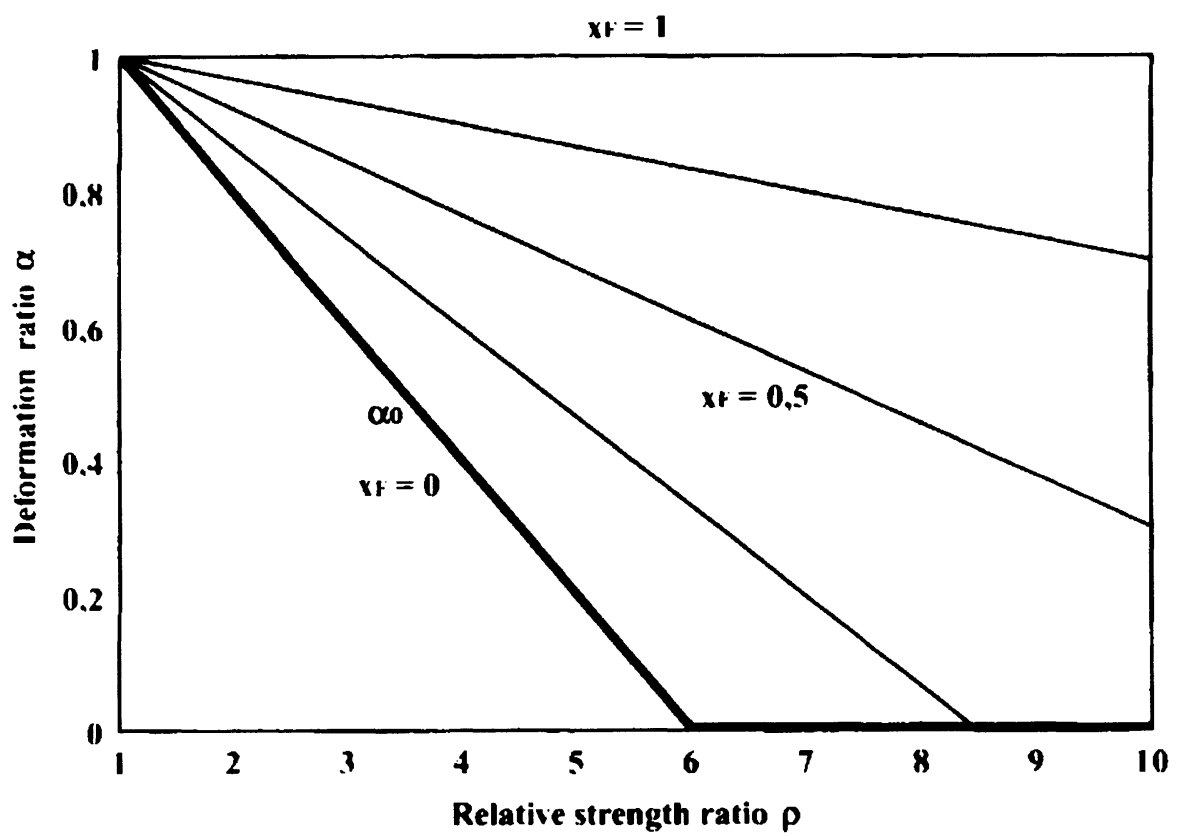

Figure 1 The deformation ratio $\alpha$ as a function of the relative yield stress $\rho$ according to eq (36), (38), (43). 


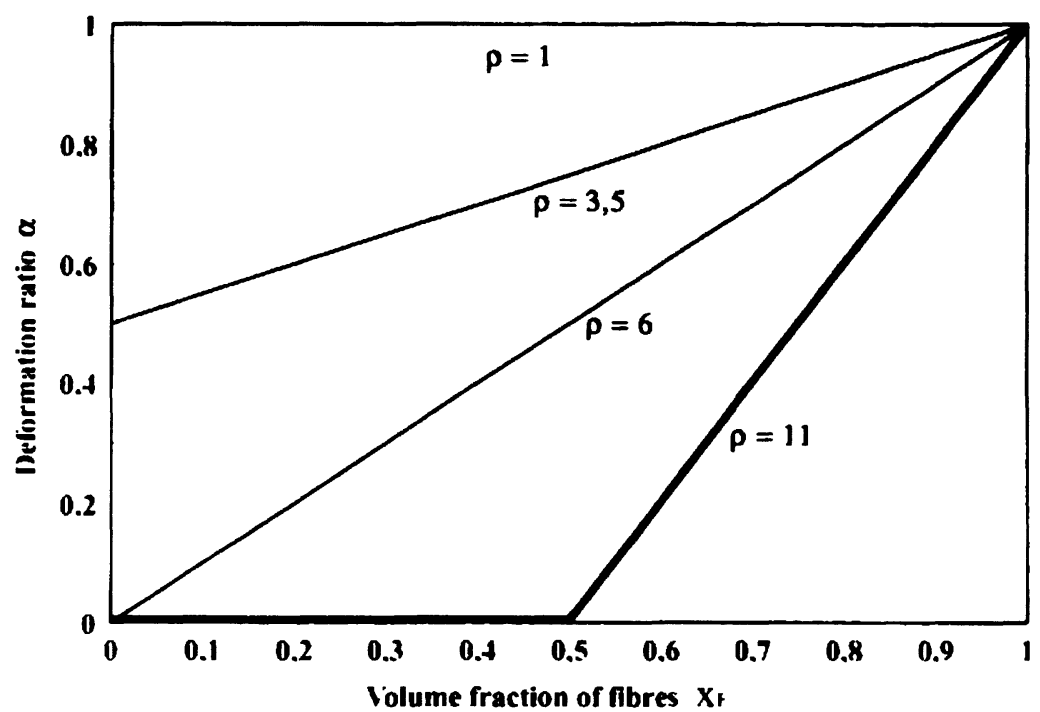

Figure 2 The deformation ratio $\alpha$ as a function of the volume fraction $x_{F}$ of the fibres according to eq (36), (38), (43).

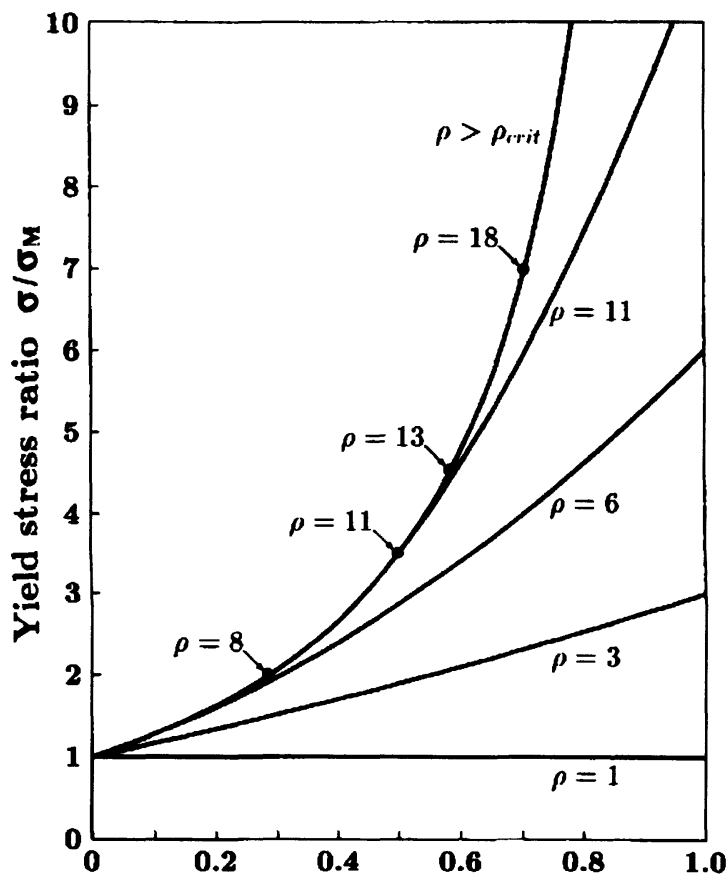

Volume fraction of fibres $x_{F}$

Figure 3 The yield stress ratio $\sigma / \sigma_{\mathrm{M}}$ of the composite compared with the matrix as a function of the volume fraction $x_{F}$ of the fibres according to eq (41), (42), (43). 


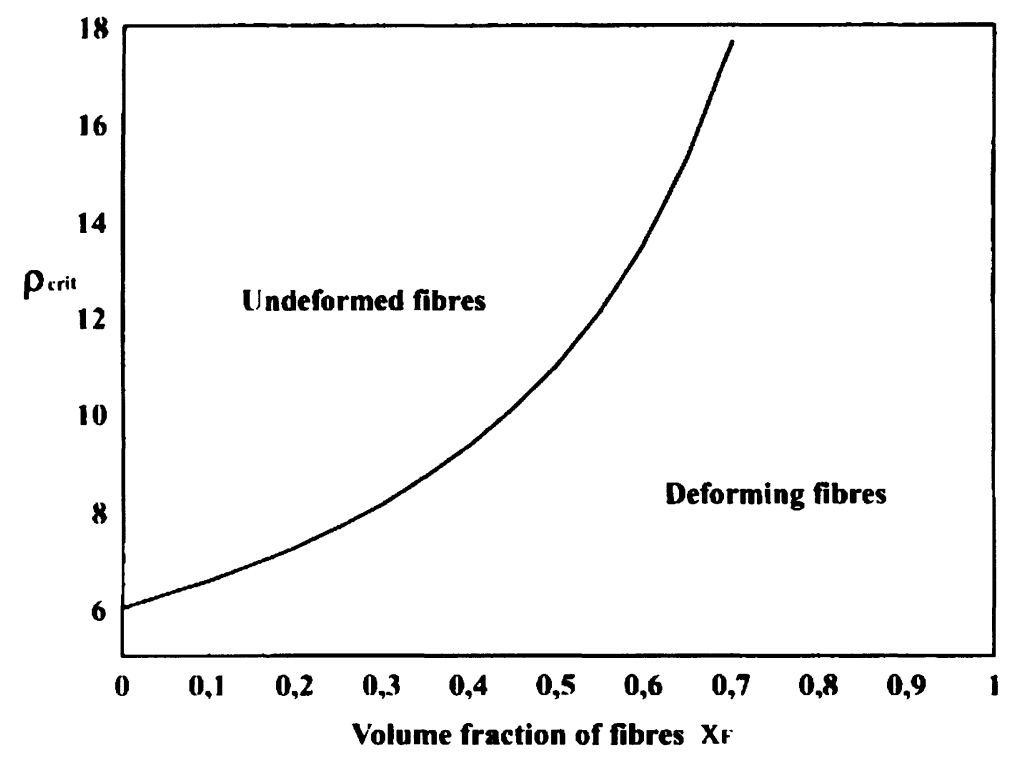

Figure 4 The critical value $\rho_{\text {crit }}$ above which the fibres remain undeformed according to eq (39), (43).

\section{COMPARISON WITH EXPERIMENTAL DATA}

In eq (2), (4) the strains $\varepsilon$ and $\varepsilon_{F}$ of the composite and the fibres respectively are defined by infinitesimally small elongations

$$
\varepsilon=\frac{d l}{l}
$$

where $l$ is the length of a fibre or the composite sample respectively. Comparison with experimental data must be done with finite deformation degrees. Hence, it is convenient to introduce the finite deformation degree $\varphi$

$$
\varphi=\int_{l_{0}}^{l_{e}} \frac{d l}{l}=\ln \frac{l_{e}}{l_{0}}=\ln \frac{1}{1-\eta}
$$

where $l_{0}$ and $l_{e}$ are the lengths of the composite or the fibres at the beginning and at the end of deformation. The logarithmic deformation degree $\varphi$ is related to the conventional deformation degree $\eta$ as is also given eq (45). With these definitions, and if we assume that $\alpha$ in eq (5) is constant during the whole deformation process, eq (5) can be written in integral form

$$
\varphi_{F}=\alpha \cdot \varphi \quad ; \quad \ln \left(1-\eta_{F}\right)=\alpha \cdot \ln (1-\eta)
$$




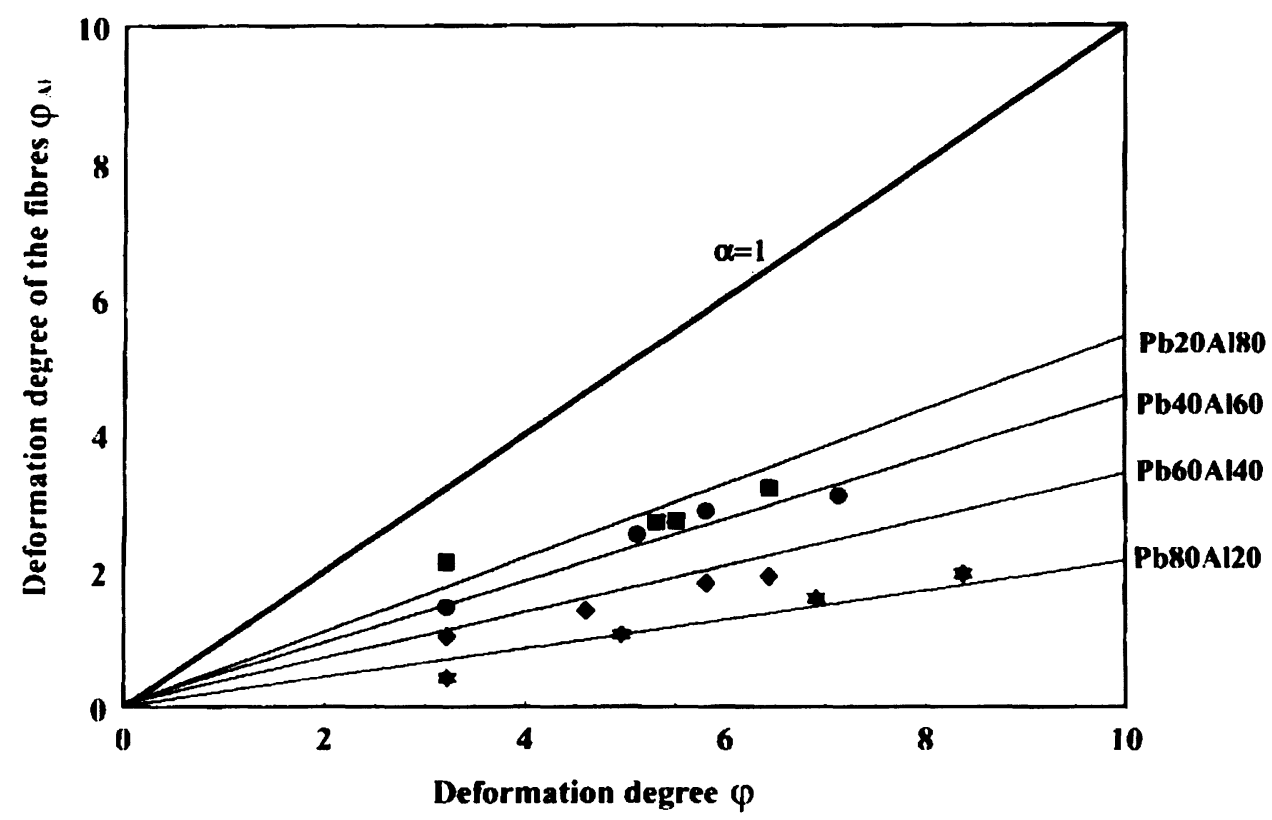

Figure 5 The deformation degree $\varphi_{\mathrm{Al}}$ of $\mathrm{Al}$ fibres in a $\mathrm{Pb}$ matrix as a function of the deformation degree $\varphi$ of the composite.

This is shown in Figure 5 for composites $\mathrm{Pb}-\mathrm{Al}$ with different volume fractions of Al-fibres. It is seen from Figure 5 that $\alpha=$ const. is a good approximation in this case.

In Figure $6, \alpha$-values of various composites are plotted as a function of the volume fraction $x_{F}$ of fibres. It is seen that in some cases the straight-line behaviour of Figure 2 is fulfilled but in others it is not. For instance, $\mathrm{Pb}-\mathrm{Zn}$ and $\mathrm{Pb}-\mathrm{Al}$ have nearly the same yield stress ratio $\rho$ but quite different $\alpha\left(x_{F}\right)$-curves. The corresponding $\alpha_{0}$-values for $x_{F} \rightarrow 0$ are, however, in good agreement. Hence, it may be assumed that deviations from the present model are particularly to be expected at higher volume fractions of fibres. The extrapolated values, $\alpha_{0}$ are plotted in Figure 7 as a function of the yield stress ratio $\rho$. It is seen that most of all systems fit quite well the corresponding curve of Figure 1. There was, however, one strong deviation, i.e. the system $\mathrm{Pb}-\mathrm{Sn}$ (not shown in the figure). Experimentally measured flow stresses of some composites during extrusion are shown in Figure 8 as a function of the volume fraction of fibres. It is seen that the experimental values are in good agreement with the curves of Figure 3.

Figure 9 finally shows the degree of texture developed in the harder Al-fibres of $\mathrm{Pb}-\mathrm{Al}$ composites. In Figure $9 \mathrm{a}$ the maximum orientation density of the Al-phase is plotted versus $\mathrm{Al}$-volume fraction at constant macroscopic deformation degree $\eta=96 \%$. In Figure $9 \mathrm{~b}$ these values are plotted versus the fibre deformation $\varphi_{\mathrm{Al}}$ taken from Figure 5. It is seen that this curve increases continuously with increasing internal deformation degree, thus corroborating the hypotheses of homogeneous deformation inside the harder fibres. 


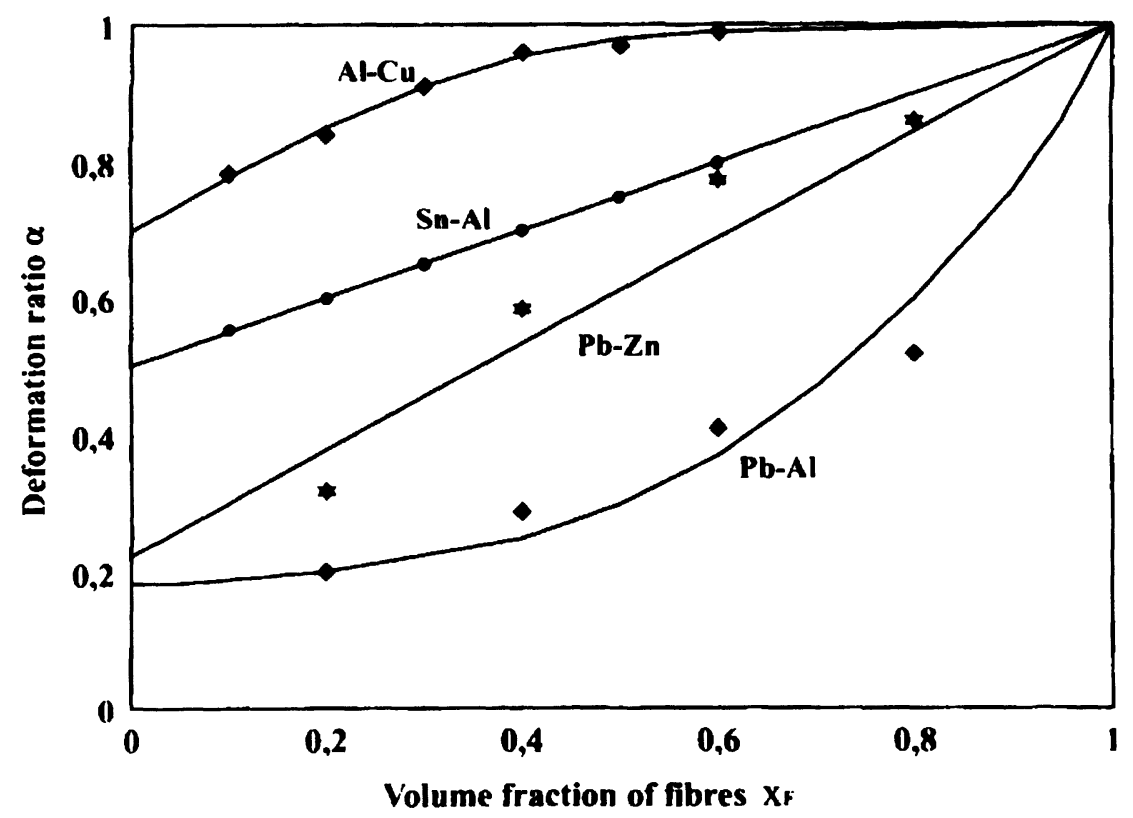

Figure 6 The deformation ratio $\alpha$ of various composites as a function of the volume fraction $x_{F}$ of the fibres.

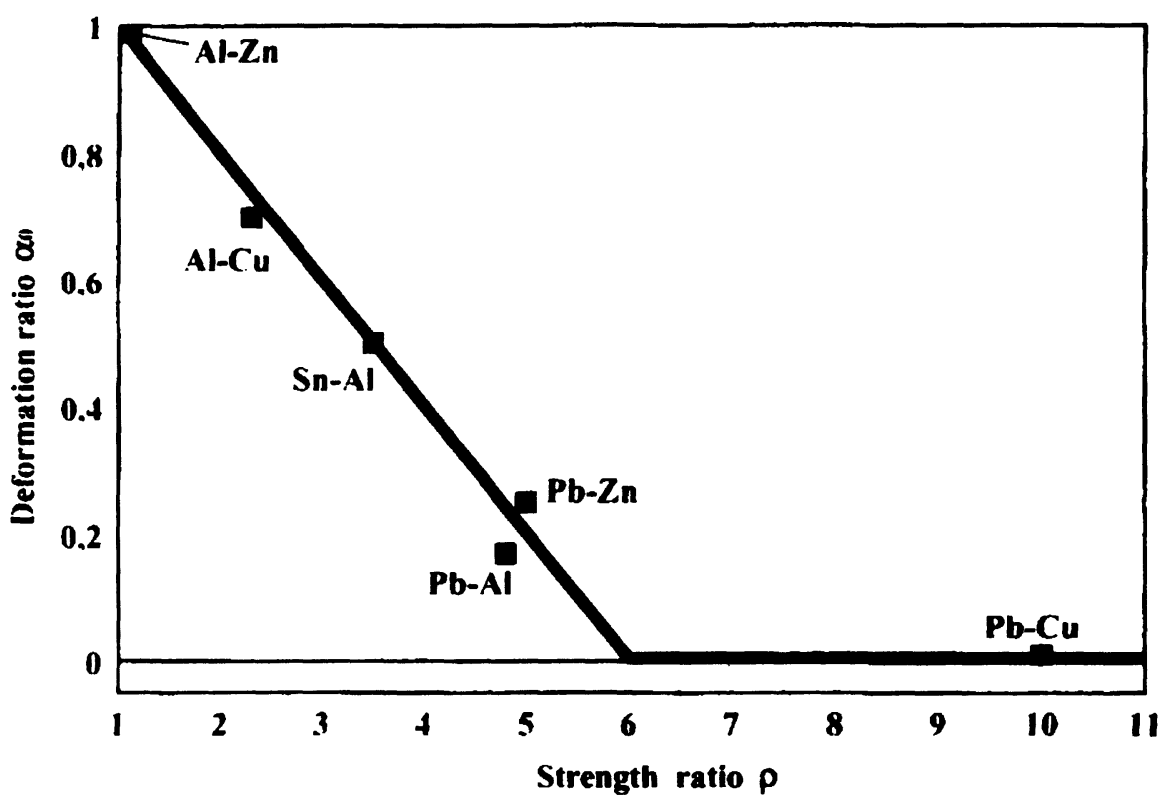

Figure 7 The deformation ratio $\alpha_{0}$ of several composites as a function of the relative yield stress $p$. 


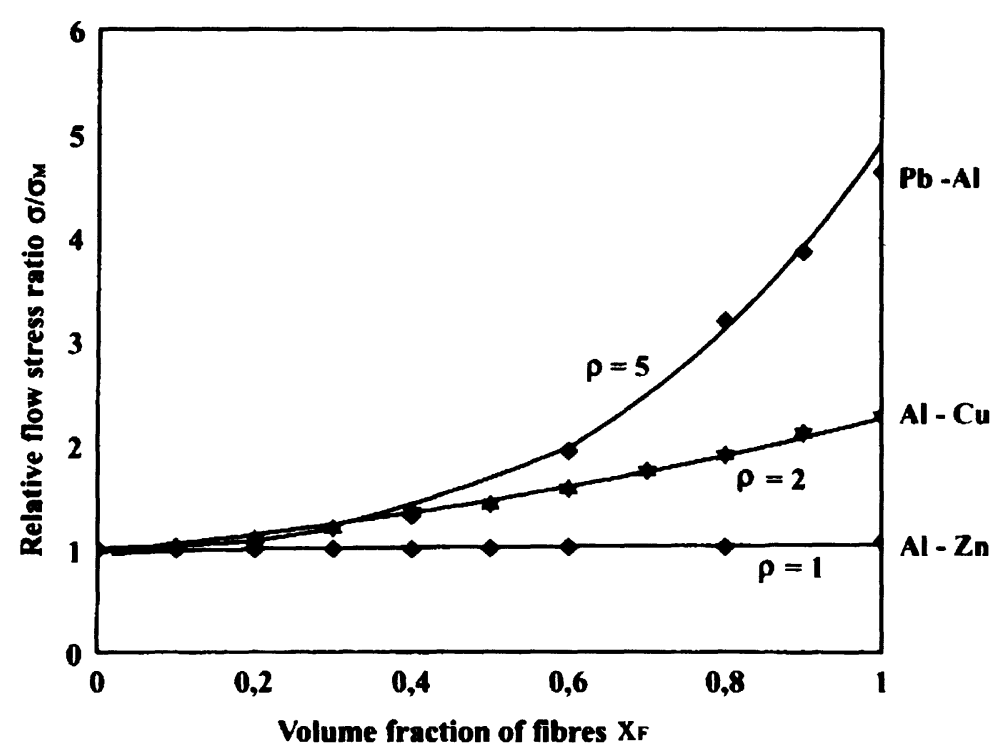

Figure 8 The relative flow stress ratio $\sigma / \sigma_{M}$ of some composites as a function of the volume fraction $\mathrm{x}_{\mathrm{F}}$ of the fibres.

\section{DISCUSSION OF THE RESULTS}

In the present calculations, a rather simple model of plastic deformation of two-phase materials is assumed. Deformation is taken to be macro-homogeneous in the composite as a whole and micro-homogeneous in the fibres. Particlularly this last assumption is only a rough approximation. The mechanical properties of matrix and fibre are characterized by single flow stress values $\sigma_{M}$ and $\sigma_{F}$ respectively and it is assumed that the ratio $\sigma_{F} / \sigma_{M}$ remains constant during deformation. Hence, differences in strain hardening as well as anisotropy due to texture formation are not taken into consideration. In the present investigations, composites with fcc matrix and bcc fibres showing extreme curling effects were not included. Nevertheless, also the composites investigated in this study showed an increasing non-circular cross section of the fibres with increasing deformation. Hence, the assumption of axially symmetric deformation of the fibres eq (4) was definitely violated. This effect is, however, a second order effect compared to the axial elongation of the fibres such that eq (4) is justified as a fist approximation. Stress and strain distribution in the softer matrix is assumed to be inhomogeneous. These inhomogeneities enter the final equations in the form of an inhomogeneity parameter $\emptyset$ eq (25). This factor is very difficult to determine, taking the stochastic distribution of the phase particles with repect to size, shape, arrangement as well as local crystal orientation into account. The quantity $\varnothing$ as a function of the volume fraction $x_{F}$ of fibres (respectively the function $\chi\left(x_{F}\right)$ eq (34)) is thus considered as a free parameter of the model which must be adapted to the experimental results.

In the limiting case of very low concentration of fibres $x_{F} \rightarrow 0$ the assumption $\chi_{0}=2.5$ describes the majority of experimental results quite satisfactorily. However, one strong deviation was found in the system $\mathrm{Pb}-\mathrm{Sn}$. This is presently being investigated in more detail. Furthermore, the assumed proportionality of $\varnothing\left(x_{F}\right)$ to $x_{F}$ and $x_{M}$ eq (34) 

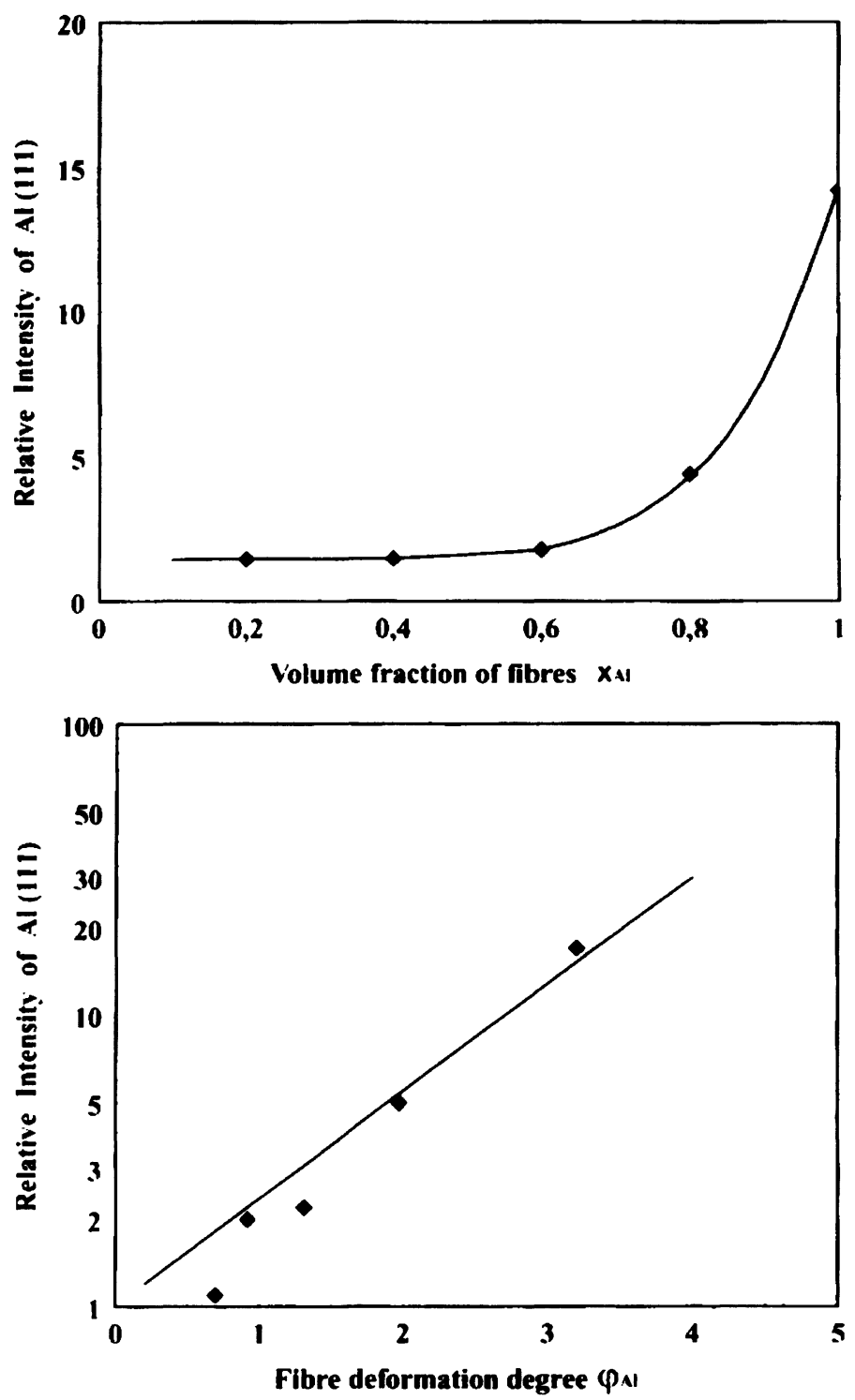

Figure 9 Deformation texture of the harder $\mathrm{Al}$ fibres in $\mathrm{PbAl}$ composites after $96 \%$ deformation: a) Relative intensity of $\mathrm{Al}_{(111)}$ as a function of composition,

b) Relative intensity of $\mathrm{Al}_{(111)}^{(111)}$ as a function of the fibre deformation degree $\varphi_{\mathrm{Al}}$.

seems to be a good approximation for some composite systems e.g. $\mathrm{Al}-\mathrm{Zn}, \mathrm{Sn}-\mathrm{Al}$ and $\mathrm{Pb}-\mathrm{Zn}$, Figure 6. The deviations of other systems such as $\mathrm{Pb}-\mathrm{Al}$ and $\mathrm{Pb}-\mathrm{Sn}$ are probably attributed to deviations from $\chi\left(\mathrm{x}_{\mathrm{F}}\right)=$ const.. The assumption $\chi\left(x_{F}\right)=$ const. is also contained in the curves Figure 3 which are compared with experimental data in Figure 8. Particularly the system $\mathrm{Pb}-\mathrm{Al}$ which deviates in Figure 6 is well represented 
in Figure 8. It must, however, be kept in mind that the accuracy of these measurements is not very high (see e.g. Böcker 1992).

Figure 5 illustrates that - within the limits of experimental accuracy - the deformation ratio $\alpha$ of the fibres compared with the macroscopic deformation is constant during the whole deformation process. From these results we may conclude that the ratio of flow stresses $\sigma_{F} / \sigma_{M}$ of both materials, fibre and matrix, remains nearly constant although the absolute values of both these quantities increase considerably.

Hence, it can be concluded that the present model is suitable to describe plastic deformation of two-pahse materials in a first approximation. The model allows to estimate quite reasonably the degree of participation of the fibres in the macroscopic deformation.

Particluarly, it gives the limit above which the fibres remain undeformed. The experimentally observed deviations give some clues in which way the model should be improved.

\section{References}

1. Ankem, S. and Margolin, H. (1986). Modelling deformation in two-phase alloys, Journ. of Metals., $38,25-29$.

2. Böcker, W. (1992). Verformungsverhalten zweiphasiger metallischer Verbundwerkstoffe, Ph D. Theses Clausthal.

3. Böcker, W. and Bunge, H. J. (1988a). Quantitative Gefügeparameter der metallischen in-SituVerbundwerkstoffe $\mathrm{Al}-\mathrm{Zn}$ und $\mathrm{Pb}-\mathrm{Al}$, Metall, 42, 466-471.

4. Böcker, W. and Bunge, H. J. (1988b). Verformungsverhalten zweiphasiger metallischer Verbundwerkstoffe. In: Verbundwerkstoffe und Werkstoffverbunde, Ed. G. Ondracek, DGM Informationsgesellschaft Oberursel, 183-188.

5. Canova, G. R., Wenk, H. R. and Molinari, A. (1992). Deformation modelling of multiphase polycrystals. Case of a Quartz-Mica aggregate, Acta Met., 40, 1519-1530.

6. Cho, K. and Gurland, J. (1988). The law of mixtures applied to the plastic deformation of twophase alloys of coarse microstructure, Metallurg. Trans., A19, 2027-2040.

7. Durand, L. and Pastor, P. (1992). Finite element method applied to tensile deformation of an equivolume two-phase alloy, Materials Characterization, 29, 39-47.

8. Fischmeister, H. and Karlsson, B. (1977). Plastizitätseigenschaften grob-zweiphasiger Werkstoffe, Z. Metallkunde, 68, 311-327.

9. Frommeyer, G. (1976). Eigenschaftsanomalien höchstverformter Silber-Kupfer-Verbunddrähte, Z.f. Werkstoff-technik, 7, 145-153.

10. Funkenbusch, P. D., Lee, J. K. and Courtney, T. H. (1987). Ductile two-phase alloys: prediction of strengthening at high strains, Metallurg. Trans., A18, 1249-1250.

11. Lian, J., Jiang, Z. and Liu, J. (1991). Theoretical model for the tensile work hardening behaviour of dual-phase steel, Mat. Sci. Eng., A147, 55-66.

12. Liesner, C. W. and Wassermann, G. (1969). Aufbau und mechanische Eigenschaften pulvermetallurgisch hergestellter Eisen-Kupfer-Legierungen, Metall, 23, 414-417.

13. McHugh, D. E., Asaro, R. J. and Shih, C. F. (1993). Computational modelling of metal matrix composite materials, Acta Metall. Mater., 41, 1461-1510.

14. Neti, S., Vijayshankar, M. N. and Ankem S. (1991). Finite element method modelling of deformation behaviour of two-phase materials, Mat. Sci. Eng., A145, 47-64.

15. Poech, M. H. and Fischmeister, H. (1992). Deformation of two-phase materials. A model based on strain compatibility, Acta Met., 40, 487-494.

16. Wahl, H. P. and Wassermann, G. (1979). Anomalien der Eigenschaften dünner Drähte aus EisenSilber-Legierungen, Z. Metallkunde, 61, 326-339.

17. Wassermann, G. (1976). Verhalten und Eigenschaften hochverformter Verbundwerkstoffe mit metallischer Matrix-Möglichkeiten der Kombination von Stoffen durch hohe und höchste Verformung, Z.f. Werkstofftechnik, 7, 125-128.

18. Werner, E. and Stüwe, H.-P. (1985). Über die Verfestigung zweiphasiger Gefüge, Z. Metallkunde, 76, 353-357. 